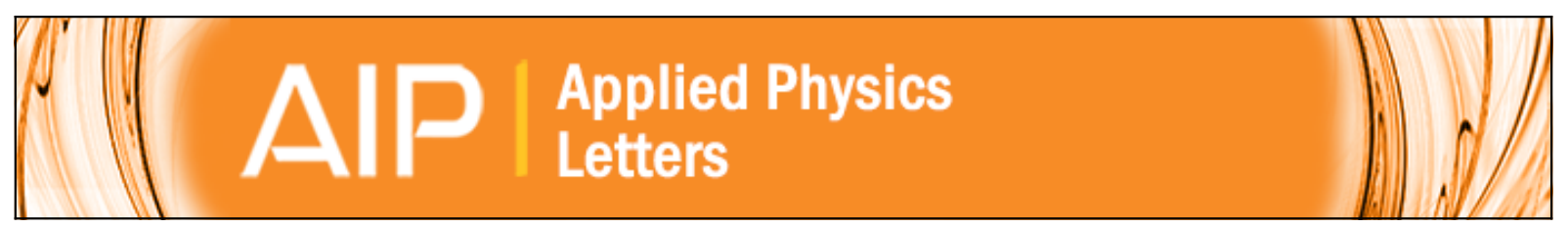

\title{
A low-temperature order-disorder transition in Cu2ZnSnS4 thin films
}

Jonathan J. S. Scragg, Léo Choubrac, Alain Lafond, Tove Ericson, and Charlotte Platzer-Björkman

Citation: Applied Physics Letters 104, 041911 (2014); doi: 10.1063/1.4863685

View online: http://dx.doi.org/10.1063/1.4863685

View Table of Contents: http://scitation.aip.org/content/aip/journal/apl/104/4?ver=pdfcov

Published by the AIP Publishing

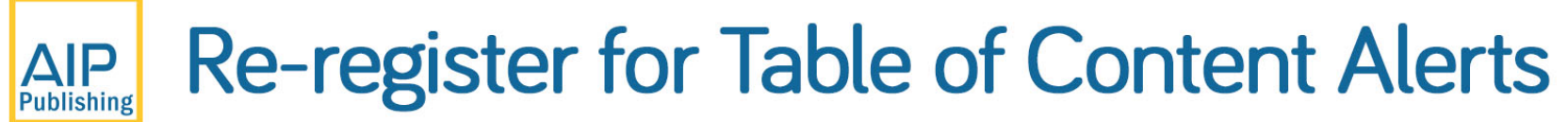




\title{
A low-temperature order-disorder transition in $\mathrm{Cu}_{2} \mathrm{ZnSnS}_{4}$ thin films
}

\author{
Jonathan J. S. Scragg, ${ }^{1, a}$ ) Léo Choubrac, ${ }^{2}$ Alain Lafond, ${ }^{2}$ Tove Ericson, ${ }^{1}$ \\ and Charlotte Platzer-Björkman ${ }^{1}$ \\ ${ }^{1}$ Angström Solar Center, Solid State Electronics, Uppsala University, Box 534, SE-751 21 Uppsala, Sweden \\ ${ }^{2}$ Institut des Matériaux Jean Rouxel (IMN), Université de Nantes, CNRS 2, rue de la Houssinière, \\ BP 32229, 44322 Nantes cedex 03, France
}

(Received 2 December 2013; accepted 16 January 2014; published online 31 January 2014)

\begin{abstract}
$\mathrm{Cu}_{2} \mathrm{ZnSnS}_{4}$ (CZTS) is an interesting material for sustainable photovoltaics, but efficiencies are limited by the low open-circuit voltage. A possible cause of this is disorder among the $\mathrm{Cu}$ and $\mathrm{Zn}$ cations, a phenomenon which is difficult to detect by standard techniques. We show that this issue can be overcome using near-resonant Raman scattering, which lets us estimate a critical temperature of $533 \pm 10 \mathrm{~K}$ for the transition between ordered and disordered CZTS. These findings have deep significance for the synthesis of high-quality material, and pave the way for quantitative investigation of the impact of disorder on the performance of CZTS-based solar cells. ( 2014 AIP Publishing LLC. [http://dx.doi.org/10.1063/1.4863685]
\end{abstract}

The attractive prospect of efficient thin film solar cells based on earth-abundant and low-toxicity elements could be realised by the use of $\mathrm{Cu}_{2} \mathrm{ZnSn}(\mathrm{S}, \mathrm{Se})_{4}$ as an alternative to the In-containing $\mathrm{Cu}(\mathrm{In}, \mathrm{Ga}) \mathrm{Se}_{2}$ absorber layer. ${ }^{1}$ Promising power conversion efficiencies of $12.6 \%$ were recently demonstrated for $\mathrm{Cu}_{2} \mathrm{ZnSn}(\mathrm{S}, \mathrm{Se})_{4},{ }^{2}$ but there is room for improvement. One troubling phenomenon is the low open circuit voltage compared to the band gap. ${ }^{3}$ A plausible reason for this is a reduction in effective band gap due to inhomogeneities in structure, phase, or composition. ${ }^{4}$ Disorder of $\mathrm{Cu}$ and $\mathrm{Zn}$ in the crystal structure is one such inhomogeneity, known to occur in synthetic material but very difficult to measure in thin films. In this paper, we show how $\mathrm{Cu} / \mathrm{Zn}$ disorder in the sulfide variant $\mathrm{Cu}_{2} \mathrm{ZnSnS}_{4}$ (CZTS) can be measured in thin films using near-resonant Raman scattering, and we use this tool to determine the critical temperature for the order-disorder transition. Using this information, synthesis methods can be designed to minimise disorder in CZTS, hopefully eliminating one source of performance-limiting inhomogeneity.

Natural specimens of CZTS were shown to adopt the kesterite structure (space group $I \overline{4}$ ) in which the $z=1 / 4$ and $z=3 / 4$ planes contain $\mathrm{Cu}$ and $\mathrm{Zn}$ ordered at the $2 c$ and $2 d$ Wyckoff positions. ${ }^{5}$ However, perfect $\mathrm{Cu} / \mathrm{Zn}$ ordering in these planes has not been observed in synthetic samples. Neutron diffraction showed that CZTS powders cooled rapidly from their synthesis temperature exhibited complete randomisation of $\mathrm{Cu}$ and $\mathrm{Zn}$ among the $2 c$ and $2 d$ sitesresulting in an effective $I \overline{4} 2 \mathrm{~m}$ space group-whilst in samples cooled at a rate of $1 \mathrm{~K} / \mathrm{h}$, about $70 \%$ of the $\mathrm{Cu}$ and $\mathrm{Zn}$ atoms were on the "correct" crystallographic sites. ${ }^{6,7}$ Even $70 \%$ ordering corresponds to large numbers of $\mathrm{Cu} / \mathrm{Zn}$ antisite defects, which are calculated to cause band gap variations on the order of $100 \mathrm{meV} .^{8}$ Fluctuations on this scale could explain a large part of the aforementioned voltage deficit. ${ }^{4,9}$ Thus, it is necessary to control $\mathrm{Cu} / \mathrm{Zn}$ disorder in CZTS, which requires knowledge of the kinetics and temperature-

\footnotetext{
${ }^{\text {a) }}$ Author to whom correspondence should be addressed. Electronic mail: jonathan.scragg@angstrom.uu.se
}

dependence of the order-disorder transition. Additionally, it is important to be able to quantify $\mathrm{Cu} / \mathrm{Zn}$ disorder in the type of thin film samples used for solar cells, which has historically been difficult.

The classic description of order-disorder transitions in crystalline materials was made by Bragg and Williams, who described the crystal as being in dynamic equilibrium, with thermal fluctuations inducing interchange of atoms between lattice sites with small energetic differences. ${ }^{10}$ Whilst the perfectly ordered structure is the ground state, the entropy associated with introduction of disorder acts in opposition, leading to a temperature-dependent, equilibrium level of ordering. Ordering is quantified by an "order parameter," which is determined by the proportion of atoms located on the correct lattice sites in the relevant substructure. The order parameter, $S$, is equal to 1 for perfect ordering, and is 0 for complete disorder. As an illustration, the model of Vineyard was used to calculate an equilibrium amount of order, $S_{\text {eqm }}$, as a function of temperature, using arbitrary parameters. ${ }^{11}$ The result is shown by the dashed line in Figure 1. The exact shape depends on the input parameters, but the behaviour is qualitatively the same in all cases: As temperature is increased, $S_{\text {eqm }}$ drops, falling rapidly to zero at a certain "critical temperature," $T_{c}$. Note that this is a second order (continuous) phase transition, so a finite amount of disorder is always present for $T>0 \mathrm{~K}$. The solid curves in Figure 1 illustrate the effect of annealing a sample with an initially low (non-equilibrium) level of order, $S_{0}$, for two different time periods $t_{1}$ and $t_{2}\left(t_{2}>t_{1}\right)$. Annealing at low temperatures (regime $I$ in the figure) does not increase $S$, despite the large perturbation from equilibrium, because the rate of atom exchange is negligible. At intermediate temperatures, regime II, thermal energy is sufficient for atomic motion, allowing $S$ to increase towards $S_{\text {eqm }}$. Since $S_{\text {eqm }}$ itself drops quickly near $T_{\mathrm{c}}$, annealing at temperatures near this value will cause a reduction in ordering compared to $S_{0}$ (regime III). For infinite anneal times, $S_{\text {eqm }}$ will be reached at all temperatures. In reality, however, there may be an upper limit on the level of order that can be obtained for practical annealing times. 


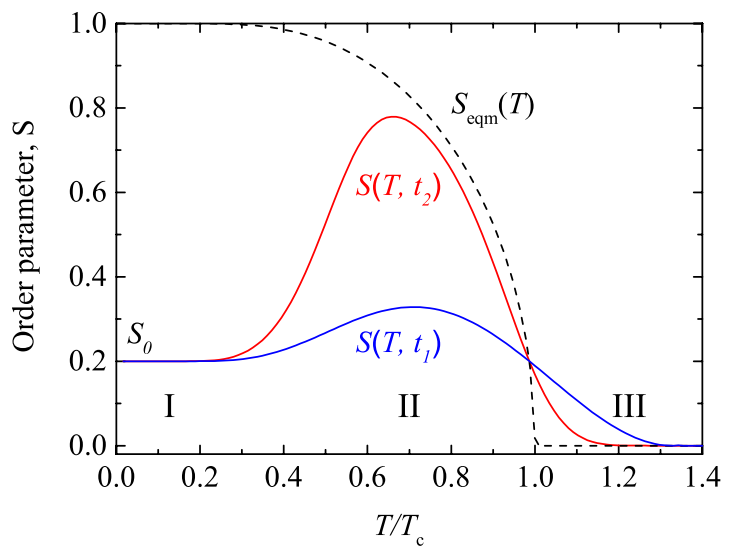

FIG. 1. The degree of order, $S$, in a material having an order-disorder transition with critical temperature $T_{\mathrm{c}}$ (calculated using the model of Vineyard. ${ }^{11}$ ) The dashed line indicates the equilibrium case (i.e., after a long anneal). The solid lines show the effect of annealing initially disordered samples for fixed time periods $t_{1}$ and $t_{2}\left(t_{2}>t_{1}\right)$.

Order parameters are ideally obtained from diffraction methods, but standard X-ray techniques cannot distinguish $\mathrm{Cu}^{+}$and $\mathrm{Zn}^{2+}$, and neutron diffraction is unsuitable for thin films. Nuclear Magnetic Resonance (NMR), which has also been shown to be sensitive to $\mathrm{Cu}-\mathrm{Zn}$ disorder in CZTS powders, ${ }^{12}$ has the same limitation. Therefore, we investigated the potential of Raman spectroscopy to assess $\mathrm{Cu} / \mathrm{Zn}$ disorder. The Raman spectrum depends on the symmetry of the crystal structure, the strength of the chemical bonds and the masses and charges of the constituent elements. It is sensitive to the local atomic environment (of the order of several coordination shells from a given central atom), ${ }^{13}$ so in principle Raman can be used to determine an order parameter. However, one must also bear in mind the influence of other factors, such as sample composition and excitation intensity. $^{14}$

We first considered two stoichiometric CZTS powder reference samples, described elsewhere. ${ }^{12}$ The samples differed only in the rate of cooling from their synthesis temperature, $1020 \mathrm{~K}$. Sample VS was slowly cooled, at a rate of $10 \mathrm{~K} / \mathrm{h}$, whilst sample VF was quenched in ice-water. NMR measurements showed that VS has a high level of order (although is not thought to be perfectly ordered), whilst VF is disordered. ${ }^{12}$ Resonant XRD on a single crystal confirmed that VF has a completely random distribution of $\mathrm{Cu}$ and $\mathrm{Zn}$ in the $z=1 / 4$ and $z=3 / 4$ planes. ${ }^{15}$

Raman spectra of VS and VF were recorded in a Renishaw inVia system using $532 \mathrm{~nm}$ and $785 \mathrm{~nm}$ laser excitation sources. The spot diameter was 5-10 $\mu \mathrm{m}$. Calibration was performed using the $\mathrm{Si}$ main mode at $520 \mathrm{~cm}^{-1}$. The laser intensity was kept low to avoid damaging the sample. ${ }^{14}$ All Raman spectra were fitted using a basis set of 11 symmetrical Lorentzian peaks derived from measurements with excitation wavelengths of 532, 633, and $785 \mathrm{~nm}$. All peaks correspond to reported kesterite modes. ${ }^{16}$ Figure 2 shows spectra of VS and VF for $532 \mathrm{~nm}$ and $785 \mathrm{~nm}$ excitation wavelengths.

For both excitation wavelengths, sample VF gives a general broadening, explained by the reduction in phonon correlation length caused by disorder. ${ }^{13}$ Beside this, in the $532 \mathrm{~nm}$ spectrum, fitting for VF revealed a small peak at

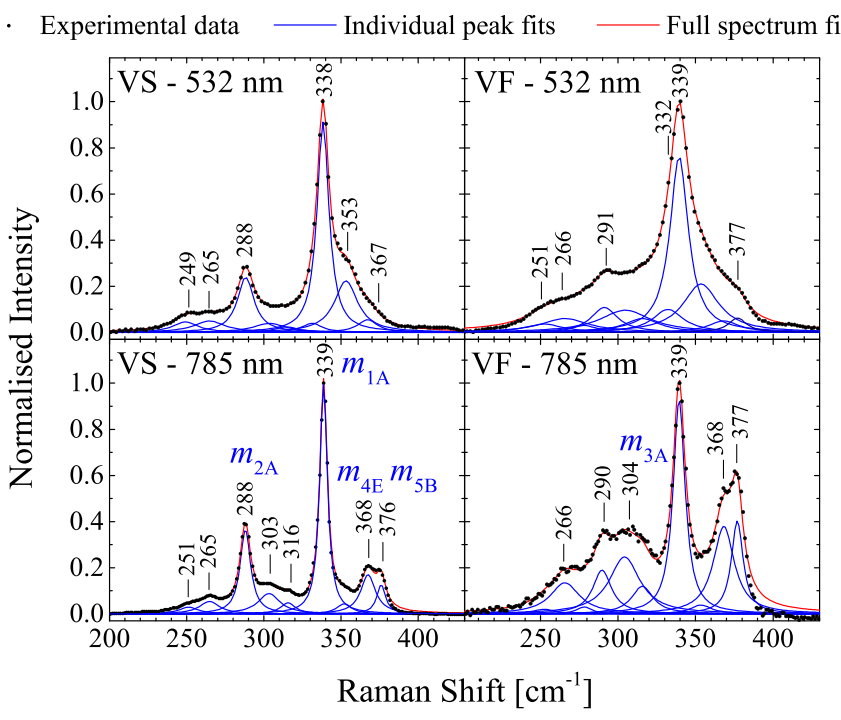

FIG. 2. Raman spectra with peak fitting for stoichiometric CZTS powder reference samples VS (slowly cooled, ordered) and VF (rapidly cooled, disordered), recorded with excitation wavelengths of 532 and $785 \mathrm{~nm}$. The spectra are normalised to the main A mode intensity.

$332 \mathrm{~cm}^{-1}$. This has been tentatively attributed to $\mathrm{Cu} / \mathrm{Zn}$ disorder, ${ }^{17,18}$ but quantitative analysis is difficult because of the overlap and variable asymmetry of the dominating A mode at $\sim 339 \mathrm{~cm}^{-1}$. In the $785 \mathrm{~nm}$ spectra, the differences between VS and VF are more dramatic. For the slowly cooled sample, the second A mode at $\sim 288 \mathrm{~cm}^{-1}$ (labelled $m_{2 \mathrm{~A}}$ ) has roughly double the intensity of the $\mathrm{E}$ and $\mathrm{B}$ modes at 368 and $376 \mathrm{~cm}^{-1}$ (labelled $m_{4 \mathrm{E}}$ and $m_{5 \mathrm{~B}}$ ). In the fast cooled sample, both of the main A modes $m_{1 \mathrm{~A}}$ and $m_{2 \mathrm{~A}}$ drop significantly relative to the rest of the spectrum, so that $m_{2 \mathrm{~A}}$ becomes similar in intensity to the previously minor A mode at $304 \mathrm{~cm}^{-1}\left(m_{3 \mathrm{~A}}\right)$, whilst $m_{4 \mathrm{E}}$ and $m_{5 \mathrm{~B}}$ have much higher relative intensity. A possible explanation for these variations $^{19}$ is that the near-resonant conditions with the $785 \mathrm{~nm}$ excitation wavelength make the spectrum sensitive to small changes in band gap, occurring as a result of antisite disorder. $^{8}$

Next, we analysed CZTS thin films made by reactive sputtering of $\mathrm{CuSn}$ alloy and $\mathrm{Zn}$ in $\mathrm{H}_{2} \mathrm{~S}$, followed by annealing in sulfur vapour at $833 \mathrm{~K}$, as described elsewhere. ${ }^{20}$ The sample composition was $\mathrm{Zn}$ rich $(\mathrm{Cu} / \mathrm{Sn}=1.9, \mathrm{Zn} / \mathrm{Sn}=1.2)$, comparable to that used for efficient solar cells. ${ }^{1}$ The films are highly uniform and Raman spectra recorded at different positions are reproducible. The spectrum of the as-prepared film using a $785 \mathrm{~nm}$ excitation is shown in Figure 3. It is intermediate to the spectra of VS and VF-for example, mode $m_{2 \mathrm{~A}}$ is clearly resolvable but is less intense than $m_{4 \mathrm{E}}$ and $m_{5 \mathrm{~B}}$ - but more closely resembles VF. This is reasonable based on the rather fast cooling used (about $30 \mathrm{~K} / \mathrm{min}$ ).

The changes in the $785 \mathrm{~nm}$ spectra were measured in two ways. First, the quantity $Q=I\left(m_{2 \mathrm{~A}}\right) / I\left(m_{3 \mathrm{~A}}\right)$ was defined, where $I(m)$ is the intensity of the indicated Raman mode based on full spectrum fitting. $Q$ is equal to $4.15,0.77$, and 1.12 for, respectively, VS, VF, and the as-prepared thin film sample. With this definition, $Q$ is larger for more ordered samples. Second, we recorded the full width at half maximum (FWHM) of the main A mode $\left(m_{1 \mathrm{~A}}\right)$, which should increase for more disordered samples. Supposing that $Q$ is 


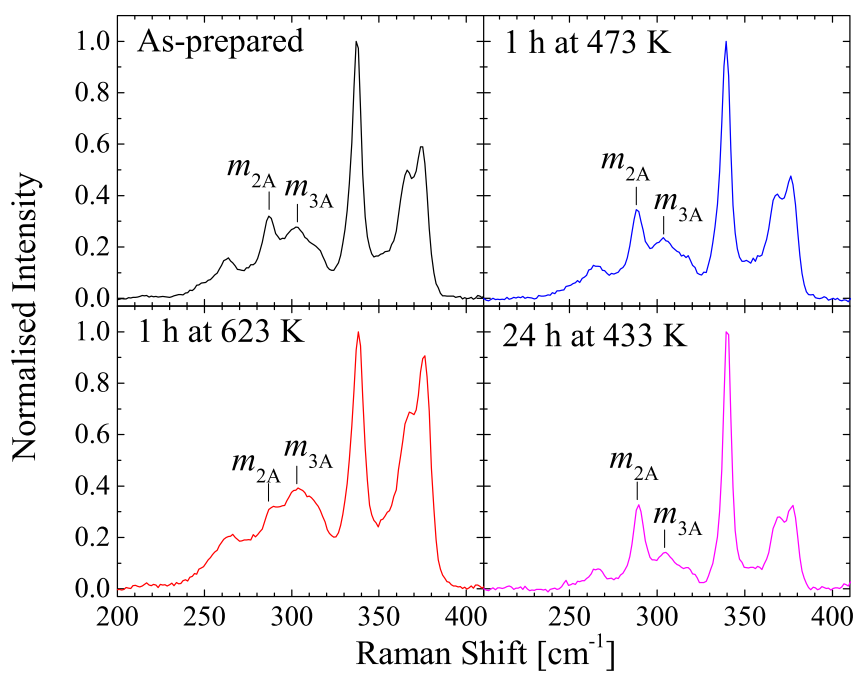

FIG. 3. Raman spectra using $785 \mathrm{~nm}$ excitation, for an as-prepared CZTS film and three sections of the same film that were post annealed as indicated. The spectra are normalised to the main A mode intensity.

related to $\mathrm{Cu} / \mathrm{Zn}$ disorder, it should vary with post-annealing in a similar way to the order parameter in Figure 1. To test this, we divided the as-prepared CZTS film into multiple identical pieces upon which we performed post-annealing in the range $373-623 \mathrm{~K}$, for either 1 or $24 \mathrm{~h}$, using $\sim 150 \mathrm{~K} / \mathrm{min}$ heating and cooling rates. The post-anneals were carried out in an atmosphere of $\mathrm{Ar}$ or $\mathrm{N}_{2}$ at $\sim 1 \mathrm{~atm}$. Spectra for some post-annealed samples are shown in Figure 3. The differences in the spectra are consistent with the differences between VS and VF.

The effect of post annealing temperature on $Q$ is plotted in Figure 4(a). There is little change in $Q$ at the lowest anneal

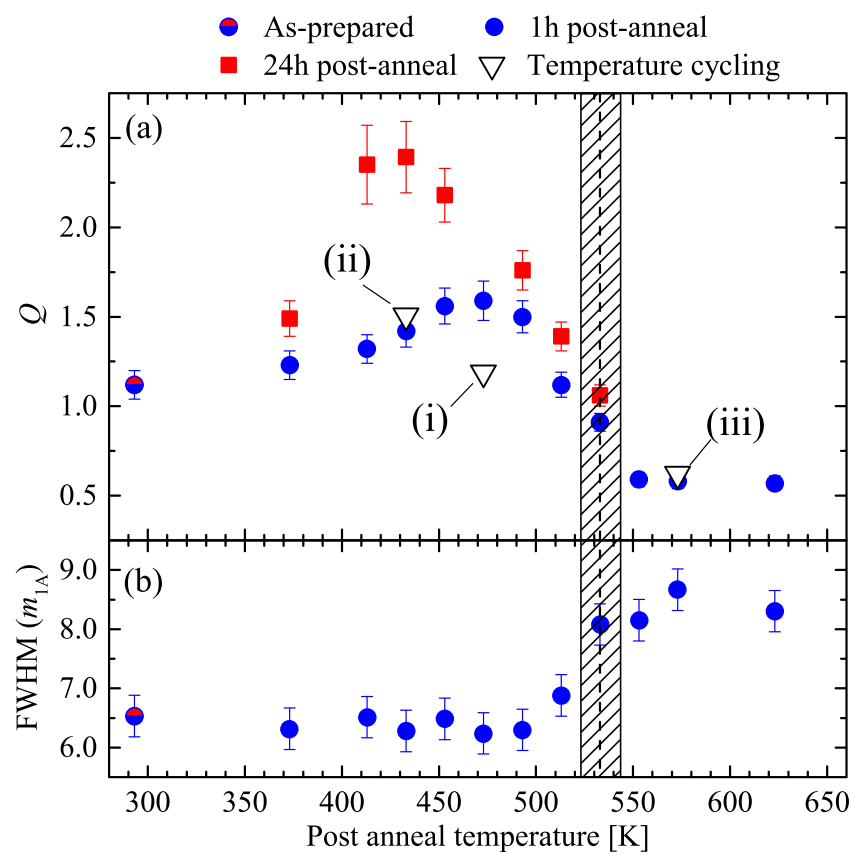

FIG. 4. (a) Variation in $Q$ for initially identical CZTS films post-annealed for different time periods. The triangles show the effect of temperature cycling on a single sample to test the reversibility of changes in $Q$. The shaded region indicates the probable location of the critical temperature $T_{\mathrm{c}}$. (b) The full-width-at-half-maximum (FWHM) of the main Raman A mode for the $1 \mathrm{~h}$ post-annealed samples. temperatures, a notable increase at intermediate temperatures $(433-493 \mathrm{~K})$, and a rapid decrease to a constant level at higher temperatures. Annealing for $24 \mathrm{~h}$ as opposed to $1 \mathrm{~h}$ considerably enhanced $Q$ for the intermediate temperatures. Figure 4(a) displays a striking similarity to Figure 1, strengthening the hypothesis that $Q$ is a function of disorder. Further support comes from the sudden increase in the FWHM of the main A mode that occurs together with the drop in $Q$, Figure 4(b): an independent measure also indicating a decrease in ordering. Interchange of the similarly sized $\mathrm{Cu}$ and $\mathrm{Zn}$ cations could plausibly occur in the temperature range over which $Q$ varies. Meanwhile, other thermally activated phenomena, e.g., grain growth or decomposition, are insignificant under these conditions. ${ }^{21}$ Indeed, no secondary phases were detected by Raman, whilst scanning electron microscopy (SEM) and energy dispersive spectroscopy (EDS) measurements (not shown) detected no changes in morphology or composition.

Reversibility is an important feature of order-disorder transitions, meaning that it should be possible to increase and decrease $Q$ for the same sample by repeated annealing at different temperatures. We tested this using the sample postannealed for $1 \mathrm{~h}$ at $573 \mathrm{~K}$. We annealed the sample for three further one-hour periods, at $473 \mathrm{~K}, 433 \mathrm{~K}$, and again at $573 \mathrm{~K}$, recording spectra between each treatment. The results are shown by the points labelled (i), (ii), and (iii) in Figure 4(a), and clearly demonstrate the expected reversibility. Note that point (i) is lower than for the direct treatment at the same temperature (the filled circle). This hysteresis is common in order-disorder transitions with slow kinetics, ${ }^{10}$ and arises from the lower initial $Q$-value for anneal (i) compared to the direct anneal.

Based on the $Q$ values, the powder VS was much more ordered even than the most-ordered thin film sample. This could be due in part to the higher $\mathrm{Zn}$ content in the thin film samples. Non-stoichiometry in the bulk phase is intimately connected to the extent of ordering, because excess $\mathrm{Zn}$ atoms must occupy non- $\mathrm{Zn}$ lattice sites (the replacement of $\mathrm{Cu}$ at a $2 c$ position is most likely ${ }^{22,23}$ ). Perfect order then becomes impossible, and the equilibrium degree of order is reduced for all temperatures. ${ }^{24}$ Also, $Q$ for VF was higher than for the most-disordered film sample: $Q$ cannot, therefore, be a function of disorder alone. This fits with our tentative explanation that variations in $Q$ arise from band gap changes: The presence of excess $\mathrm{Zn}_{\mathrm{Cu}}$ sites would presumably affect the band gap in a similar manner to antisite disorder. Therefore, bulk phase composition must be considered when using $Q$ to compare different samples.

The detailed agreement with expectations from theory suggest that $Q$ can indeed be used to track $\mathrm{Cu} / \mathrm{Zn}$ disorder in thin film CZTS. Quantification of disorder, as well as investigation of the causes of the spectral changes, will be the subject of future work. The most important outcome at present is that we can use Figure 4 to estimate $T_{\mathrm{c}}$, the critical temperature for $\mathrm{Cu} / \mathrm{Zn}$ disorder. $Q$ drops below the as-prepared value at $\sim 523 \mathrm{~K}$, and reaches a lower limit at $533-553 \mathrm{~K}$. Therefore, a reasonable estimate for $T_{\mathrm{c}}$ is between these values; we suggest a figure of $533 \pm 10 \mathrm{~K}\left(260 \pm 10^{\circ} \mathrm{C}\right)$.

$\mathrm{Cu} / \mathrm{Zn}$ disorder is expected to cause the unit cell to expand, ${ }^{25}$ primarily by an increase in the $c$ parameter. ${ }^{12}$ 
Thus, on top of normal thermal expansion, a further increase in $c$ is predicted to occur in the region of $T_{\mathrm{c}}$. This phenomenon has indeed been observed experimentally: Synchrotron XRD measurements with in situ heating on $70 \%$ ordered kesterite revealed an anomalous expansion of the $c$-parameter starting at $\sim 460 \mathrm{~K}$ and ending at $\sim 530 \mathrm{~K} .^{26}$ In the light of our results, the existence and temperature range of this anomaly can be fully explained by a gradual loss of order that reaches completion at the critical temperature. Our estimate for $T_{\mathrm{c}}$ is thus consistent with independent findings.

The implications of the low critical temperature are significant. CZTS is normally grown at $720-830 \mathrm{~K}$, far above the critical temperature. Our results indicate that at these growth temperatures, ordered $I \overline{4}$ kesterite cannot exist; the fully disordered, $I \overline{4} 2 \mathrm{~m}$ kesterite will form instead. Only when the temperature drops below about $533 \mathrm{~K}$, i.e., during post-synthesis cooling, can ordering among $\mathrm{Cu}$ and $\mathrm{Zn}$ begin. Due to the second order nature of the transition, the amount of disorder remaining at room temperature is likely to be relatively large. The exact extent of ordering by the end of the synthesis will depend only on the part of the cooling that occurs below $533 \mathrm{~K}\left(260^{\circ} \mathrm{C}\right)$. This low-temperature part of the cooling is rarely reported. Nevertheless, it is apparently critical for determining the level of disorder, or, in other words, the concentration of $\mathrm{Cu}_{\mathrm{Zn}}$ and $\mathrm{Zn}_{\mathrm{Cu}}$ antisite defects, in CZTS. Since such defects are a possible cause of potential fluctuations, ${ }^{9}$ we arrive at the interesting conclusion that the electrical properties of CZTS could be modified by relatively low temperature processes. Possible effects on device characteristics must be fully explored in the light of these findings.

In summary, near-resonant Raman was shown to be a convenient tool to track $\mathrm{Cu} / \mathrm{Zn}$ disorder in CZTS films, allowing determination of the critical temperature for the transition from ordered to disordered kesterite as a relatively low $533 \pm 10 \mathrm{~K}\left(260 \pm 10^{\circ} \mathrm{C}\right)$. With this information, it is now possible to design synthesis methods that minimise disorder in CZTS. Future studies will focus on Raman-based quantification of disorder, as well as the predicted link between $\mathrm{Cu} / \mathrm{Zn}$ disorder and voltage losses in CZTS solar cells.

This work was funded by the Swedish Research Council and the Swedish Energy Agency (Uppsala), and the French ANR under Grant NovACEZ (ANR-10-HABISOL-008) (Nantes). The purchase of the Raman system at Uppsala was partially financed by a generous donation from the Knut \& Alice Wallenberg Foundation. Finally, J.S. would like to thank Viktor Izquierdo-Roca of the Catalonia Institute for Energy Research, Barcelona, for valuable conversations regarding Raman spectroscopy and the likely origin of the changes in $Q$.

${ }^{1}$ D. B. Mitzi, O. Gunawan, T. K. Todorov, and D. A. R. Barkhouse, Philos. Trans. R. Soc., A 371(1996), 20110432 (2013).

${ }^{2}$ W. Wang, M. T. Winkler, O. Gunawan, T. Gokmen, T. K. Todorov, Y. Zhu, and D. B. Mitzi, "Device Characteristics of CZTSSe Thin-Film Solar Cells with 12.6\% Efficiency," Adv. Energy Mater. (2013).

${ }^{3}$ S. Siebentritt and S. Schorr, Prog. Photovoltaics 20(5), 512 (2012).

${ }^{4}$ U. Rau and J. H. Werner, Appl. Phys. Lett. 84(19), 3735 (2004).

${ }^{5}$ S. R. Hall, J. T. Szymanski, and J. M. Stewart, Can. Mineral. 16(2), 131 (1978).

${ }^{6}$ S. Schorr, Sol. Energy Mater. Sol. Cells 95(6), 1482 (2011).

${ }^{7}$ S. Schorr, H.-J. Hoebler, and M. Tovar, Eur. J. Mineral. 19(1), 65 (2007).

${ }^{8}$ D. Huang and C. Persson, Thin Solid Films 535(0), 265 (2013).

${ }^{9}$ T. Gokmen, O. Gunawan, T. K. Todorov, and D. B. Mitzi, Appl. Phys. Lett. 103(10), 103506 (2013).

${ }^{10}$ W. L. Bragg and E. J. Williams, Proc. R. Soc. London. Ser A 145(855), 699 (1934).

${ }^{11}$ G. H. Vineyard, Phys. Rev. 102(4), 981 (1956).

${ }^{12}$ L. Choubrac, M. Paris, A. Lafond, C. Guillot-Deudon, X. Rocquefelte, and S. Jobic, Phys. Chem. Chem. Phys. 15(26), 10722 (2013).

${ }^{13}$ G. Gouadec and P. Colomban, Prog. Cryst. Growth Mater. 53(1), 1 (2007).

${ }^{14}$ M. Y. Valakh, V. M. Dzhagan, I. S. Babichuk, X. Fontane, A. PerezRodriquez, and S. Schorr, JETP Lett. 98(5), 255 (2013).

${ }^{15}$ A. Lafond, L. Choubrac, C. Guillot-Deudon, P. Fertey, M. Evain, and S. Jobic, "X-ray resonant single crystal diffraction technique, a powerful tool to investigate the kesterite structure of the photovoltaic $\mathrm{Cu}_{2} \mathrm{ZnSnS}_{4}$ compound," Acta Crystallographica B (unpublished).

${ }^{16}$ A. Khare, B. Himmetoglu, M. Johnson, D. J. Norris, M. Cococcioni, and E. S. Aydil, J. Appl. Phys. 111(8), 083707 (2012).

${ }^{17}$ M. Grossberg, J. Krustok, J. Raudoja, and T. Raadik, Appl. Phys. Lett. 101(10), 102102 (2012).

${ }^{18}$ M. Y. Valakh, O. F. Kolomys, S. S. Ponomaryov, V. O. Yukhymchuk, I. S. Babichuk, V. Izquierdo-Roca, E. Saucedo, A. Perez-Rodriguez, J. R. Morante, S. Schorr, and I. V. Bodnar, Phys. Status Solidi (RRL) 7(4), 258 (2013).

${ }^{19}$ V. Izquierdo-Roca, private communication (2013).

${ }^{20}$ J. J. Scragg, T. Kubart, J. T. Wätjen, T. Ericson, M. K. Linnarsson, and C. Platzer-Björkman, Chem. Mater. 25(15), 3162 (2013).

${ }^{21}$ J. J. Scragg, T. Ericson, T. Kubart, M. Edoff, and C. Platzer-Björkman, Chem. Mater. 23(20), 4625-4633 (2011).

${ }^{22}$ L. Choubrac, A. Lafond, C. Guillot-Deudon, Y. Moëlo, and S. Jobic, Inorg. Chem. 51(6), 3346 (2012).

${ }^{23}$ A. Lafond, L. Choubrac, C. Guillot-Deudon, P. Deniard, and S. Jobic, Z. Anorg. Allg. Chem. 638(15), 2571 (2012).

${ }^{24}$ G. J. Dienes, Acta Metall. 3(6), 549 (1955).

${ }^{25}$ S. Chen, X. G. Gong, A. Walsh, and S.-H. Wei, Appl. Phys. Lett. 94(4), 041903 (2009).

${ }^{26}$ S. Schorr and G. Gonzalez-Aviles, Physica Status Solidi A 206(5), 1054 (2009). 